\title{
Prevalence of Fibromyalgia Syndrome and Its Correlations with Arrhythmia in Patients with Palpitations
}

\author{
Ayhan Aşkın ${ }^{1, *}$, Ece Güvendi², Ayten Özkan³, Ersin Çağrı Şimşek, Uğur Kocabaş̧, Aliye Tosun ${ }^{1}$
}

\begin{abstract}
Objective: It is aimed to determine the prevalence of fibromyalgia syndrome (FMS) and its correlations with arrhythmia in patients with palpitations.

Material and Methods: Sixty-two patients who underwent electrophysiological study (EPS) due to palpitation complaints in Cardiology department and 40 healthy controls were included in the study. The precise diagnosis of arrhythmia was established using EPS. All participants were screened for FMS using American College of Rheumatology 2010 Fibromyalgia diagnostic criteria. Clinical assessments included measurement of severity of pain, fatigue and morning fatigue with visual analog scale (VAS), functional status with Fibromyalgia Impact Questionnaire (FIQ), and anxiety/depression with Hospital Anxiety and Depression Scale (HAD).

Results: FMS was diagnosed in 22 of the 62 patients (36\%), and 4 of the 40 healthy controls $(10 \%)(p<0.05)$. Mean HAD scores of the patients were significantly higher than the controls $(p<0.05)$. The frequency of FMS was statistically higher in EPS+ and EPS- patients with palpitations than in controls $(p<0.05)(38 \%, 33 \%, 10 \%$, respectively), but there was no difference between EPS+ and EPS- groups. There were no statistical differences between the 3 groups, in terms of pain intensity, fatigue level, FIQ and HAD scores ( $p>0.05)$. EPS+ patients with FMS had higher fatigue levels, HAD and FIQ scores than EPS- patients, although statistically insignificant. HV durations were statistically longer in the EPS- subgroup $(p<0.05)$ but other EPS data were similar.

Conclusion: FMS frequency and HAD anxiety scores were found to be higher in patients with palpitation complaints. However, we found no association between arrhythmia, EPS parameters and FMS. In our clinical practice we should keep in mind to carry out assessments in terms of FMS in patients with palpitation.
\end{abstract}

KEYWORDS

fibromyalgia; arrhythmia; electrophysiological study; anxiety

AUTHOR AFFILIATIONS

${ }^{1}$ Department of Physical Medicine and Rehabilitation, Katip Çelebi University, Faculty of Medicine, Izmir, Turkey

2 Department of Physical Medicine and Rehabilitation, Katip Çelebi University Atatürk Training and Research Hospital, Izmir, Turkey

${ }^{3}$ Department of Physical Medicine and Rehabilitation, Selçuk State Hospital, Izmir, Turkey

${ }^{4}$ Department of Cardiology, University of Health Science, Tepecik Training and Research Hospital, Izmir, Turkey

${ }^{5}$ Department of Cardiology, Katip Çelebi University Atatürk Training and Research Hospital, Izmir, Turkey

* Corresponding author: Katip Çelebi Üniversitesi Atatürk Eğitim ve Araştırma Hastanesi AMATEM binası FTR Ek Servisi, Karabağlar/İzmir, Turkey; ayhanaskin@hotmail.com

Received: 19 July 2017

Accepted: 18 October 2017

Published online: 23 April 2018

Acta Medica (Hradec Králové) 2017; 60(4): 146-151

https://doi.org/10.14712/18059694.2018.10

(c) 2017 The Authors. This is an open-access article distributed under the terms of the Creative Commons Attribution License (http://creativecommons.org/licenses/by/4.0), which permits unrestricted use, distribution, and reproduction in any medium, provided the original author and source are credited. 


\section{INTRODUCTION}

Fibromyalgia syndrome (FMS) is a form of extraarticular or soft tissue rheumatism which is characterized with widespread pain, fatigue, sleep disturbances and cognitive disorders (1). It is more frequently seen in women within age range of 20 and 55 years. Mean prevalence of FMS was reported as $1.78 \%$ (2). Although its pathogenesis is still unclear, chronic stress and neuroendocrine abnormalities were suggested as causative factors, and it has been shown in many studies that the autonomic nervous system dysfunction plays an important role in the development of the disease (3-6).

The autonomic nervous system is a regulatory system that protects the homeostasis within the body and plays an important role in response to stress. Sympathetic/parasympathetic balance enables the continuity of the normal autonomic nervous system. As a result of sympathetic hyperactivation and/or parasympathetic dysfunction, the body may not be able to tolerate excessive stress, and it may result in sleep disorders, stiffness, tender points, and exercise intolerance $(6,7)$. Cohen et al. (8) found that autonomic dysregulation may have implications regarding the symptomatology, physical and psychological aspects of health status in FMS, while Furlan et al. (6) reported that sympathetic activity was increased by disruption of autonomic balance during rest in female patients with FMS.

Pathological alterations in the sympathetic and parasympathetic systems are known to result in various types of arrhythmias (9). Arrhythmia risk has been investigated in patients with FMS, and an increase in the autonomic activity and fluctuations in the heart rates were reported (7). However; there are conflicting results in the literature regarding relationship between arrhythmia and FMS. Doğru et al. (10) found that sympathetic activity significantly increased whereas parasympathetic activity decreased in patients with FMS which led to the changes in autonomic circadian activity. These changes caused an increase in the prevelance of arrhythmia and the ratio of supraventricular tachycardia was found as increased in patients when compared to healthy controls. Sarıfakıoğlu et al. (11) have also showed that the risk of atrial fibrillation was increased in patients with FMS. In contrast to these data, Kulshreshtha et al. (12) reported that the autonomic reflex arc and cardiac autonomic function were normal in patients with FMS. Yolbas et al. (13) also reported that long QT and P wave dispersions, which indirectly indicate atrial and/or ventricular arrhythmias, are seen in the patients with FMS with the same frequency as the controls.

Anxiety and depression are common in patients with FMS. Numerous studies suggested that accompanying anxiety results in cardiac autonomic disorders and increases the risk of coronary artery disease in healthy individuals (14). Sarıfakığlu et al. (11) did not find any relation between arrhythmia and anxiety-depression, but reported that the risk of atrial fibrillation was increased in patients with FMS. However, Zamuner et al. (15) reported that the disruption of neurocardiac integrity in FMS have an impact on pain and quality of life.
In this study, we aimed to determine the prevelance of FMS and its correlations with arrhythmia in patients with palpitations. Our secondary aim was to investigate the relations between arrhythmia, FMS and anxiety-depression.

\section{MATERIALS AND METHODS}

The study has been designed as cross-sectional study. All participants were informed about the study and their consents were obtained. The study has been performed according to the Declaration of Helsinki and approved by the ethical committee of our hospital (Approval number: 2015/100).

\section{PARTICIPANTS}

Sixty-two patients who underwent electrophysiological study due to palpitations in Cardiology department and 40 healthy controls were included in the study. Patients with previously diagnosed cardiac pathologies (hypertension, valve diseases, myocardial infarction, rheumatic heart diseases, cardiac insufficiency, cardiomyopathy, cardiac pacemakers), thyroid dysfunction, malignancy, diabetes mellitus, peripheral vascular disease, chronic lung disease, psychiatric disease, renal disease, pregnancies or using medications including beta blockers, calcium channel blockers or anti-arrhythmic drugs were not included in the study. The demographic data (age, gender, educational status, and marital status), body mass index, symptom durations, and medical histories (allergy history, medications, past operations and accompanying diseases) of all participants were recorded.

\section{CLINICAL ASSESSMENTS}

All participants were screened for FMS using American College of Rheumatology 2010 Fibromyalgia diagnostic criteria. These criteria includes the "widespread pain index" displaying the number of painful body parts and the "symptom severity scale" indicating cognitive symptoms, sleep, fatigue and somatic symptoms (1). Clinical assessments included measurement of severity of pain, fatigue and morning fatigue with visual analog scale (VAS), functional status with Fibromyalgia Impact Questionnaire (FIQ), and anxiety/depression with Hospital Anxiety and Depression Scale (HAD).

Fibromyalgia Impact Questionnaire is a specific measure that assesses the physical function and health status in patients with FMS. FIQ is composed of 10 items. In the inquiry, the first item contains a scale on which 11 daily activities are evaluated over $0-3$. The second item determines the number of days in which the patient felt well during the last week, while the third item questions the number of days in which the patient was unable to go to work or do housework due to the illness during the past week. The other seven items evaluate the severity of pain, the ability to do work, fatigue, and sleep quality, stiffness, anxiety, and depression within the last week, using VAS. The total FIQ score is maximum 100 points. High scores indicate low functionality level (16). 
Hospital Anxiety and Depression Scale is an assessment scale developed to identify the risk regarding anxiety and depression, and measures their levels and severities. Its subscales are anxiety (HAD-A) and depression (HAD-D). It contains 14 questions in total. Seven of them (odd numbers) measure anxiety and the other seven (even numbers) measure depression. The lowest and highest scores that a person can obtain from either subscale are 0 and 21, respectively (17).

\section{ELECTROPHYSIOLOGICAL STUDY (EPS)}

Electrophysiological study is an invasive procedure based on the assessment of intracardiac electrogram records. Cardiac electrical potentials are obtained from the sinus node, right atrium, atrioventricular (AV) node and the right ventricle using catheters placed as a standard (1820 ). In addition, while the intracardiac electrograms are being obtained from the inside of the heart using these catheters, stimulation can be performed as well. "PA = Intra-atrial communication time, $\mathrm{A}=$ Atrial Activity, $\mathrm{AH}=$ Atrial-his communication time, $\mathrm{H}=$ His branch activity, HV = His-ventricular communication time, V = Ventricular activity" are the values measured as a standard within the EPS procedure. The PR interval in the superficial ECG is the sum of the intervals of PA, AH and HV. Data obtained as the basal cycle length is the RR interval in the ECG. Even though these measured times may vary depending on the age and sex, normal values are considered as AH 60-125 ms and HV 35-55 ms (20). The AH interval can be affected by autonomic changes especially. Sympathetic stimulation shortens the interval, whereas parasympathetic stimulation extends it. The HV interval is not affected by autonomic changes and measurement techniques. In the assessment of atrioventricular node functions, atrial-his (AH) and his-ventricular (HV) periods are used among the basal communication times. The determination of the moment, when a tissue with the ability to transmit stimulation within the heart can no longer transmit such stimulation, is called the "refractory period measurement". The measurement of the refractory period in the atrioventricular node is called "AV Wenckebach". Even though it varies depending on the gender and age, it is considered to be below $450 \mathrm{~ms}$ as a normal value (20).

\section{STATISTICAL ANALYSIS}

SPSS 16.0 (IBM Corporation, Armonk, New York, United States) program was used to analyze the variables. The Shapiro-Wilk test was used to check whether the data is normally distributed. One-way analysis of variance (ANOVA) test was used to compare three independent groups with each other based on the quantitative data, and the Pearson Chi-Square test was used to compare categorical variables with each other. In the subgroup analysis, two independent groups were compared with each other according to the quantitative results using Mann Whitney $\mathrm{U}$ test. Correlation analysis of clinical parameters was carried out using Pearson correlation test. Quantitative variables were displayed in tables as mean \pm standard deviation (std) and median range (maximum-minimum), and categorical variables were displayed as $n(\%)$. The variables were examined at $95 \%$ confidence level and $p$ value $<0.05$ was considered significant.

\section{RESULTS}

Sixty-two patients (mean ages; $47.22 \pm 11.24$ years) with palpitations and 40 healthy controls (mean ages $45.87 \pm$ 12.40 years) were included in the study. FMS has been diagnosed in 22 patients (36\%), and in 4 controls $(10 \%)$ $(\mathrm{p}<0.05)$. EPS data yielded arrhythmias in $52 \%$ of the patients, whereas $48 \%$ had no abnormality. All the patients with positive EPS had supraventricular tachycardias. Mean HAD-anxiety scores $(6.51 \pm 4.24)$ of the patients

Tab. 1 Demographic and clinical characteristics of the patients with palpitation complaints.

\begin{tabular}{|c|c|c|c|}
\hline & \multicolumn{3}{|c|}{$\begin{array}{l}\text { Patients with Palpitation } \\
\text { complaints }(n=62)\end{array}$} \\
\hline & $\mathrm{n}$ & $\%$ & $\begin{array}{l}\text { Mean } \pm \text { Standard } \\
\text { deviation }\end{array}$ \\
\hline Age (years) & & & $47.22 \pm 11.24$ \\
\hline \multicolumn{4}{|l|}{ Gender } \\
\hline Female & 36 & 58 & \\
\hline Male & 26 & 42 & \\
\hline BMI $\left(\mathrm{kg} / \mathrm{m}^{2}\right)$ & & & $25.56 \pm 3.50$ \\
\hline Education status (years) & & & $8.01 \pm 2.93$ \\
\hline \multicolumn{4}{|l|}{ Marital status } \\
\hline Married & 45 & 73 & \\
\hline Single & 17 & 27 & \\
\hline \multicolumn{4}{|l|}{ FMS } \\
\hline Yes & 22 & 36 & \\
\hline No & 40 & 64 & \\
\hline Symptom Period (months) & & & $58.37 \pm 74.47$ \\
\hline Pain severity & & & $2.24 \pm 3.02$ \\
\hline Fatigue severity & & & $3.24 \pm 3.07$ \\
\hline Morning fatigue severity & & & $2.85 \pm 3.15$ \\
\hline FIQ & & & $20.59 \pm 14.70$ \\
\hline \multicolumn{4}{|l|}{ HAD } \\
\hline Depression & & & $4.70 \pm 4.08$ \\
\hline Anxiety & & & $6.51 \pm 4.24$ \\
\hline \multicolumn{4}{|l|}{ EPS } \\
\hline Positive & 32 & 52 & \\
\hline Negative & 30 & 48 & \\
\hline \multicolumn{4}{|l|}{ EPS Results } \\
\hline $\mathrm{BCL}$ & & & $819.12 \pm 154.05$ \\
\hline $\mathrm{AH}$ & & & $102.32 \pm 25.71$ \\
\hline HV & & & $42.96 \pm 4.36$ \\
\hline Wenckebach & & & $364.35 \pm 73.74$ \\
\hline Ventricular Refractor & & & $220.96 \pm 39.11$ \\
\hline
\end{tabular}

$\mathrm{N}$ : number, \% percentage

BMI: Body Mass Index, FMS: Fibromyalgia syndrome, FIQ:

Fibromyalgia Impact Questionnaire, HAD: Hospital Anxiety

Depression Scale, EPS: Electrophysiological Study, BCL: Basal

Cycle Length, AH: duration between Atrial-his bundle, HV: duration

between his-ventricle 
with palpitations were significantly higher than the control $(4.60 \pm 4.31)(p<0.05)$. Demographical data, clinical characteristics, and EPS results of the patients are shown in Table 1.

When the patient group was divided into two subgroups as the ones with positive EPS (EPS+) and negative EPS (EPS-); no statistical difference was found between the 3 subgroups (EPS+, EPS- and the control group) in terms of demographical characteristics $(p>0.05)$. The prevelance of FMS was statistically higher in EPS+ and EPSgroups than in controls (38\%, 33\%, 10\%, respectively) ( $\mathrm{p}$ $<0.05$ ), but there was no significant difference between EPS + and EPS- groups. There was no statistical difference between the 3 groups, in terms of pain intensity, fatigue level, FIQ and HAD scores ( $>$ > 0.05). Comparison of demographical and clinical parameters of the patients and the controls based on their EPS results has been summarized in Table 2.

When the patients with FMS were divided into two subgroups as EPS+ and EPS-, no statistically significant difference was found in-between ( $p>0.05)$, even though EPS+ FMS patients had higher pain severities, fatigue levels, HAD and FIQ scores. According to the EPS results of both subgroups, HV durations were statistically longer in the EPS- subgroup $(p<0.05)$. Other EPS data were similar. The comparison of the demographical and clinical characteristics of the patients with FMS based on the EPS results is displayed in Table 3.

No significant correlation was detected between EPS scores and pain severity, fatigue level, FIQ, HAD scores $(\mathrm{p}>0.05)$.

\section{DISCUSSION}

In our study, FMS frequency and HAD anxiety scores were found to be higher in patients with palpitations than in controls. In the EPS- FMS patients, it was found that HV times were prolonged. However, these patients showed similar characteristics with the EPS+ patients. No correlation was found between EPS results and clinical parameters.

It is known that the basic pathophysiological mechanisms in FMS are closely associated to neurohormonal disorders and autonomic dysfunction. Hypothalamic-pituitary-adrenal axis changes and the effects of sympathetic nervous system on homeostasis have been shown in FMS physiopathology (21). It is thought that autonomic dysfunction may be effective in the pathogenesis of major symptoms such as sleep disorders, stiffness, tender points, exercise intolerance $(6,7)$, and Vincent et al. (22) reported that autonomic symptoms such as dizziness, palpitation, sensitivity to sound and light, and gastrointestinal symptoms, were also frequently seen in these patients other than characteristic symptoms. Martinez-Lavin et al. (23) found that sleep disorders and fatigue were associated with increased sympathetic activation during the night period in patients with FMS, whereas Doğru et al. (10) reported increased sympathetic activation in these patients compared to the control group especially at night, and decreased parasympathetic activity. In our study we could not find statistical difference or any clinical relations between the groups, in terms of pain intensity, fatigue level, fibromyalgia symptom severity and EPS parameters.

Tab. 2 Comparison of demographic and clinical characteristics of the patients and of the controls.

\begin{tabular}{|c|c|c|c|c|c|c|c|c|c|c|}
\hline & \multicolumn{3}{|c|}{$\operatorname{EPS}(+)(n=32)$} & \multicolumn{3}{|c|}{$\operatorname{EPS}(-)(n=30)$} & \multicolumn{3}{|c|}{ Controls $(n=40)$} & \multirow[b]{2}{*}{$\mathrm{p}$} \\
\hline & $\mathrm{n}$ & $\%$ & Mean \pm Std & $n$ & $\%$ & Mean \pm Std & $\mathrm{n}$ & $\%$ & Mean \pm Std & \\
\hline Age (years) & & & $44.56 \pm 11.25$ & & & $50.06 \pm 10.69$ & & & $45.87 \pm 12.40$ & 0.152 \\
\hline Gender & & & & & & & & & & 0.104 \\
\hline Female & 22 & 72 & & 14 & 47 & & 21 & 53 & & \\
\hline Male & 10 & 28 & & 16 & 53 & & 19 & 47 & & \\
\hline BMI $\left(\mathrm{kg} / \mathrm{m}^{2}\right)$ & & & $25.20 \pm 3.94$ & & & $25.95 \pm 2.98$ & & & $24.44 \pm 2.89$ & 0.167 \\
\hline Education status (years) & & & $7.84 \pm 3.05$ & & & $8.20 \pm 2.83$ & & & $8.02 \pm 2.97$ & 0.894 \\
\hline Marital status & & & & & & & & & & 0.873 \\
\hline Married & 24 & 75 & & 21 & 70 & & 30 & 75 & & \\
\hline Single & 8 & 25 & & 9 & 30 & & 10 & 25 & & \\
\hline FMS & & & & & & & & & & $0.015^{*}$ \\
\hline Yes & 12 & 38 & & 10 & 33 & & 4 & 10 & & \\
\hline No & 20 & 62 & & 20 & 67 & & 36 & 90 & & \\
\hline Pain severity & & & $1.87 \pm 2.82$ & & & $2.63 \pm 3.23$ & & & $1.95 \pm 2.34$ & 0.494 \\
\hline Fatigue severity & & & $3.71 \pm 3.41$ & & & $2.73 \pm 2.62$ & & & $2.05 \pm 2.93$ & 0.070 \\
\hline Morning fatigue severity & & & $3.25 \pm 3.41$ & & & $2.43 \pm 2.84$ & & & $1.70 \pm 2.71$ & 0.097 \\
\hline FIQ & & & $20.84 \pm 15.28$ & & & $20.32 \pm 14.31$ & & & $17.66 \pm 14.57$ & 0.615 \\
\hline \multicolumn{11}{|l|}{ HAD } \\
\hline Depression & & & $5.50 \pm 4.66$ & & & $3.86 \pm 3.23$ & & & $3.90 \pm 3.10$ & 0.129 \\
\hline Anxiety & & & $6.53 \pm 4.77$ & & & $6.50 \pm 3.67$ & & & $4.60 \pm 4.31$ & 0.094 \\
\hline
\end{tabular}

$\mathrm{N}$ : number, \% percentage, ${ }^{*}$ statistically significant $(p<0.05)$

EPS: Electrophysiological Study, BMI: Body Mass Index, FMS: Fibromyalgia syndrome, FIQ: Fibromyalgia Impact Questionnaire, HAD:

Hospital Anxiety Depression Scale

Chi-Square test, Single variance analysis 
Tab. 3 Comparison of demographic and clinical characteristics of the patients with fibromyalgia based on the electrophysiological study results.

\begin{tabular}{|c|c|c|c|c|c|c|c|}
\hline & \multicolumn{3}{|c|}{$\operatorname{EPS}(+)(n=12)$} & \multicolumn{3}{|c|}{$\operatorname{EPS}(-)(n=10)$} & \multirow[b]{2}{*}{ p } \\
\hline & $\mathrm{n}$ & $\%$ & Median (min-max) & $\mathrm{n}$ & $\%$ & Median (min-max) & \\
\hline Age (years) & & & $45.5(32-63)$ & & & $55(53-64)$ & $0.014^{*}$ \\
\hline Gender & & & & & & & $0.015^{*}$ \\
\hline Female & 12 & 100 & & 6 & 60 & & \\
\hline Male & 0 & 0 & & 4 & 40 & & \\
\hline $\mathrm{BMI}\left(\mathrm{kg} / \mathrm{m}^{2}\right)$ & & & $27.5(19-32)$ & & & $25.3(23-34)$ & 0.146 \\
\hline Education status (years) & & & $8(2-11)$ & & & $8(3-11)$ & 0.728 \\
\hline Marital status & & & & & & & 0.364 \\
\hline Married & 9 & 75 & & 9 & 90 & & \\
\hline Single & 3 & 25 & & 1 & 10 & & \\
\hline Symptom Period (months) & & & $36(12-200)$ & & & $27(2-48)$ & 0.111 \\
\hline Pain severity & & & $4(1-10)$ & & & $7(2-9)$ & 0.059 \\
\hline Fatigue severity & & & $8(3-10)$ & & & $5(3-9)$ & 0.094 \\
\hline Morning fatigue severity & & & $7(0-10)$ & & & $6(3-10)$ & 0.547 \\
\hline FIQ & & & $37.2(18-51)$ & & & $34.2(23-57)$ & 0.999 \\
\hline \multicolumn{8}{|l|}{ HAD } \\
\hline Depression & & & $9(3-17)$ & & & $6(1-10)$ & 0.073 \\
\hline Anxiety & & & $9(4-17)$ & & & $9(0-15)$ & 0.817 \\
\hline \multicolumn{8}{|l|}{ EPS Results } \\
\hline $\mathrm{BCL}$ & & & $792(520-1030)$ & & & $875(633-1094)$ & 0.552 \\
\hline AH & & & $97(84-140)$ & & & $90(64-160)$ & 0.642 \\
\hline HV & & & $41(35-48)$ & & & $48(38-50)$ & $0.003^{*}$ \\
\hline Wenckebach & & & $320(280-420)$ & & & $320(290-550)$ & 0.842 \\
\hline Ventricular Refractor & & & $210(200-250)$ & & & $210(210-230)$ & 0.370 \\
\hline
\end{tabular}

$\mathrm{N}$ : number, \% percentage, ${ }^{*}$ statistically significant $(\mathrm{p}<0.05)$

EPS: Electrophysiological Study, BMI: Body Mass Index, FMS: Fibromyalgia syndrome, FIQ: Fibromyalgia Impact Questionnaire, HAD: Hospital Anxiety Depression Scale, BCL: Basal Cycle Length, AH: duration between atrial-his bundle, HV: duration between his-ventricle Chi-Square test, Mann Whitney U test

It was also thought that autonomic dysfunction, which is considerably important in pathogenesis, has a relation with the risk of cardiovascular disease in patients with FMS, and increased cardiac dysfunction was investigated by various studies in such patients. Meeus et al. (7) found that there is an increase in the autonomic activity and there are fluctuations regarding the stable and unstable heart rates of patients with FMS. Contrary to this, Dursun et al. (24) found that the heart rate turbulence parameters reflecting cardiac autonomic activity did not change in female patients with FMS compared to healthy controls. In our study, EPS parameters (especially AH interval) were used in order the detect autonomic changes but no significant clinical data could be identified.

Palpitation (sensation of fast or irregular heartbeat) is one of the most frequent causes of admission to the hospital, and history, physical examination, 12-lead electrocardiogram and holter recordings are most commonly used for diagnostic purposes (25). Apart from these, electrophysiological study (EPS) carried out with invasive procedures is a widely used method for determining etiology of palpitation (26). In a study conducted by Tsiachris et al. (27), EPS has been shown to be a highly reliable and advanced diagnostic method for the diagnosis of palpitation and has been shown to improve the quality of life by providing a success- ful guidance in arrhythmia mechanism and in patient management. In their study, they did not find any EPS data to explain the palpitation in only $16.6 \%$ of patients with palpitation symptoms. EPS was carried out in our study as well for etiology and diagnosis purposes, on the patients admitted with palpitation complaints and EPS data which could explain the palpitation was detected in $52 \%$ of the patients, whereas $48 \%$ of the patients had no abnormality regarding EPS. Similar to our data, in a study, in which 172 patients with palpitation symptoms but who have normal electrocardiogram data were assessed through EPS, positive EPS data has been found in $50 \%$ of the patients (28).

There is very limited data in the literature regarding relationship between arrhythmia and FMS. In our study, FMS was detected in $36 \%$ of the patients who admitted with palpitation complaints and supraventricular tachycardia was found in all EPS+ patients. In a similar study, Doğru et al. (10) which evaluated 50 individuals with FMS and 30 healthy controls, the prevalence of supraventricular extra systole was found as $48 \%$ in the FMS group and this ratio was found as $23.3 \%$ in the control group. Sar1fakıoglu et al. (11) have also showed that the risk of atrial fibrillation was increased in patients with FMS. In contrast to these data, Yolbas et al. (13) reported similar frequencies of arrhythmias in patients with FMS when com- 
pared with controls. Likewise, we also found no significant correlation between EPS scores and clinical parameters.

Cardiovascular diseases are often accompanied by anxiety and depression, and these are generally known to increase the symptoms of the disease. In the studies conducted, it has been detected that $30 \%$ of the patients admitted to the hospital due to myocardial infarction have depressive findings, and $15-20 \%$ of them have been diagnosed with major depression (29). It is also known that there is an increase in anxiety symptoms after acute cardiovascular events (30). Likewise, in our study HAD-anxiety score average was significantly higher in all patients admitted with palpitation complaints than in the control group. However, there was no statistically significant difference, even though EPS (+) patients with FMS had higher pain severities, fatigue levels, HAD and FIQ scores. The relation between anxiety-depression and cardiac events in patients with FMS has been addressed in a very few amount of the studies. Zamuner et al. (15) indicated that disruption of neurocardiac integrity in patients with FMS have an impact on pain and quality of life. In a study where the relation between anxiety, depression and arrhythmia have been investigated in 59 patients diagnosed with FMS, higher anxiety-depression scores were obtained when compared to the control group, but its relation with arrhythmia has not been suggested (11).

Our study has several limitations. First is the small number of patients and controls. Secondly, only EPS data was evaluated in our study, without using the ECG and the 24-hour Holter ECG data, which are the starting criteria of arrhythmia assessment. EPS was also not performed in the control group.

\section{CONCLUSION}

FMS frequency and HAD anxiety scores were found to be higher in patients with palpitation complaints. However, we found no association between arrhythmia, EPS parameters and FMS. In our clinical practice we should keep in mind to carry out assessments in terms of FMS in patients with palpitation. Further studies with larger sample sizes are needed to clarify the relationship between FMS and arrhythmia.

\section{REFERENCES}

1. Wolfe F, Clauw DJ, Fitzcharles MA, et al. The American College of Rheumatology preliminary diagnostic criteria for fibromyalgia and measurement of symptom severity. Arthritis Care Res (Hoboken) 2010; 62: 600-610.

2. Heidari F, Afshari M, Moosazadeh M. Prevalence of fibromyalgia in general population and patients, a systematic review and meta-analysis. Rheumatol Int 2017; 37(9): 1527-1539.

3. Masi AT. An intuitive person-centered perspective on fibromyalgia syndrome and its management. Baillieres Clin Rheumatol 1994; 8: 957-993.

4. Buskila D, Press J. Neuroendocrine mechanisms in fibromyalgia-chronic fatigue. Best Pract Res Clin Rheumatol 2001; 15: 747-758.

5. Raj SR, Brouillard D, Simpson CS, Hopman WM, Abdollah H. Dysautonomia among patients with fibromyalgia: a noninvasive assessment. J Rheumatol 2000; 27: 2660-2665.

6. Furlan R, Colombo S, Perego F, et al. Abnormalities of cardiovascular neural control and reduced orthostatic tolerance in patients with primary fibromyalgia. J Rheumatol 2005; 32: 1787-1793.
7. Meeus M, Goubert D, De Backer F, et al. Heart rate variability in patients with fibromyalgia and patients with chronic fatigue syndrome: a systematic review. Semin Arthritis Rheum 2013; 43: 279-287.

8. Cohen H, Neumann L, Shore M, Amir M, Cassuto Y, Buskila D. Autonomic dysfunction in patients with fibromyalgia: application of power spectral analysis of heart rate variability. Semin Arthritis Rheum 2000; 29: 217-227.

9. Coumel P. Cardiac arrhythmias and the autonomic nervous system. J Cardiovasc Electrophysiol 1993; 4: 338-55.

10. Doğru MT, Aydin G, Tosun A, et al. Correlations between autonomic dysfunction and circadian changes and arrhythmia prevalence in women with fibromyalgia syndrome. Anadolu Kardiyol Derg 2009; 9: 110-117.

11. Sarifakioglu B, Guzelant AY, Alpsoy S, et al. Is there a new finding added to the fibromyalgia syndrome? North Clin Istanb 2014; 1: 6-12.

12. Kulshreshtha P, Gupta R, Yadav RK, Bijlani RL, Deepak KK. A comprehensive study of autonomic dysfunction in the fibromyalgia patients. Clin Auton Res 2012; 22: 117-122.

13. Yolbaş S, Yıldırım A, Düzenci D, Karakaya B, Dağlı MN, Koca SS. QT dispersion and $\mathrm{P}$ wave dispersion in patients with fibromyalgia. Eur J Rheumatol 2016; 3: 165-168.

14. Kawachi I, Colditz GA, Ascherio A, et al. Prospective study of phobic anxiety and risk of coronary heart disease in men. Circulation 1994; 89: 1992-1997.

15. Zamunér AR, Forti M, Andrade CP, Avila MA, da Silva E. Respiratory Sinus Arrhythmia and its Association with Pain in Women with Fibromyalgia Syndrome. Pain Pract 2016; 16: 704-711.

16. Bennett RM. The Fibromyalgia Impact Questionnaire (FIQ): A review of its development, current version, operating characteristics and uses. Clin Exp Rheumatol 2005; 23: 154-162.

17. Zigmond AS, Snaith PR. The hospital anxiety and depression scale. Acta Psychiatr Scand 1983; 67: 361-370.

18. Tracy CM, Akhtar M, DiMarco JP, et al. American College of Cardiology/American Heart Association 2006 update of the clinical competence statement on invasive electrophysiology studies, catheter ablation, and cardioversion: a report of the American College of Cardiology/American Heart Association/American College of Physicians Task Force on Clinical Competence and Training: developed in collaboration with the Heart Rhythm Society. Circulation 2006; 114: 1654-1668.

19. Stevenson WG, Soejima K. Recording techniques for clinical electrophysiology. J Cardiovasc Electrophysiol 2005; 16: 1017-1022.

20. Rankin AC, Quinn FR, Rae AP. Clinical Cardiac Electrophysiology. In: Peter W. Macfarlane, A. van Oosterom, Olle Pahlm, Paul Kligfield, Michiel Janse, John Camm, editors. Comprehensive Electrocardiology. Springer-Verlag London Limited, 2011, p. 1133-1161.

21. Tanrıverdi F, Karaca Z, Unluhızarcı K, Keleştimur F. The hypothalamo-pituitary-adrenal axis in chronic fatigue syndrome and fibromyalgia syndrome. Stress 2007; 10: 13-25.

22. Vincent A, McAllister SJ, Singer W, et al. A report of the autonomic symptom profile in patients with fibromyalgia. J Clin Rheumatol 2014; 20: 106-108.

23. Martinez-Lavin M, Hermosillo AG, Rosas M, Soto ME. Circadian studies of autonomic nervous balance in patients with fibromyalgia: a heart rate variability analysis. Arthritis Rheum 1998; 41: 19661971.

24. Dursun H, Onrat E, Ercan E, et al. Heart rate turbulence analysis in female patients with fibromyalgia. Clinics (Sao Paulo) 2015; 70: 296-300.

25. Raviele A, Giada F, Bergfeldt L, et al. European Heart Rhythm Association. Management of patients with palpitations: a position paper from the European Heart Rhythm Association. Europace 2011; 13: 920-934.

26. Abbott AV. Diagnostic approach to palpitations. Am Fam Physician 2005; 71: 743-750.

27. Tsiachris D, Koutagiar I, Gatzoulis KA, et al. Diagnosis and management of phantom tachycardias based on an electrophysiologically guided approach. Hellenic J Cardiol 2016; 57: 340-344.

28. Vallès E, Martí-Almor J, Bazan V, et al. Diagnostic and prognostic value of electrophysiologic study in patients with nondocumented palpitations. Am J Cardiol 2011; 107: 1333-1337.

29. Myers V, Gerber Y, Benyamini Y, Goldbourt U, Drory Y. Post-myocardial infarction depression: increased hospital admissions and reduced adoption of secondary prevention measures - a longitudinal study. J Psychosom Res 2012; 72: 5-10.

30. Lane D, Carroll D, Ring C, Beevers DG, Lip GY. The prevalence and persistence of depression and anxiety following myocardial infarction. Br J Health Psychol 2002; 7: 11-21. 